\title{
Digital subtraction angiography in patients with cerebral ischaemic attacks and normal continuous wave doppler studies
}

\author{
M GUIDOTTI, ${ }^{*}$ G LANDI, ${ }^{*}$ G SCOTTI, $\dagger$ G SCARLATO* \\ From the Neurological Clinic II, University of Milan and the Department of Neuroradiology, Niguarda \\ Hospital, Milan, Italy.
}

SUMMARY The cervico-cranial arteries were studied using digital subtraction angiography in 50 patients with reversible cerebral ischaemic attacks and normal continuous wave Doppler examination. Digital subtraction angiography was able to visualise the arteries satisfactorily in 45 patients $(90 \%)$, and revealed minor arterial abnormalities in 12 patients $(24 \%)$. However, neither occlusions nor stenoses greater than $50 \%$ of the diameter of the vessel lumen were found. Although digital subtraction angiography is more accurate than continuous wave Doppler examination in detecting arterial lesions, it did not provide alternative therapeutic choices in any of these patients.

In recent decades the morphological study of the major neck vessels has acquired new importance as a means of assessing risk of stroke in patients with reversible ischaemic attacks. ${ }^{2}$ Cerebral angiography is still the accepted method for detection of cervico-cerebral atherosclerosis. Nonetheless, because of the risk of morbidity and mortality which it entails, ${ }^{34}$ other less invasive methods for the examination of the neck arteries have been developed. Continuous wave Doppler arterial recording has become particularly common for screening cerebrovascular disease. It is, however, recognised that lesions causing stenosis of less than $50 \%$ of the vascular lumen cannot be detected with continuous wave Doppler, and that ulcerated lesions do not show up if not associated with a significant stenotic lesion. ${ }^{56}$

In recent years intravenous digital subtraction angiography, which allows a fairly sensitive simultaneous visualisation of the four cervico-cerebral arterial trunks, has gained in importance. ${ }^{7-10}$ The advantages and limitations of this technique in relation to conventional angiography have been described. ${ }^{1-13}$ However, the superiority of digital

Address for reprint requests: Dr Mario Guidotti, Clinica Neurologica III, Ospedale Policlinico, via F. Sforza 35, 20122Milano-Italy.

Received 3 April 1984 and in revised form 7 June 1984. Accepted 25 June 1984 subtraction angiography and its usefulness in the management of patients with normal continuous wave Doppler examination have not been assessed, and this was the aim of our study.

\section{Patients and methods}

Fifty patients referred to our Neurological Department between May 1982 and June 1983 for partially or completely reversible ischaemic attacks had normal continuous wave Doppler sonography of the cervical vessels.

Digital subtraction angiography was carried out in all cases within 15 days of the continuous wave Doppler examination. The diagnosis of cerebral ischaemic attacks was made on the grounds of clinical history, neurological examination and CT scan. No patients had ever had surgery for cerebrovascular disease.

Continuous wave Doppler recording of the neck vessels was performed using a Parks model 1010 bidirectional instrument, by transcutaneous scanning of the neck with a focused 4.2 and $9.6 \mathrm{MHz}$ Doppler probe attached to a position-sensing armature. All continuous wave Doppler studies were performed by the same clinician. The examination included insonnation of the periorbital branches of the ophthalmic arteries, as well as direct insonnation of the common, external and internal carotid arteries. Its accuracy has been recently confirmed by Trockel et al. ${ }^{14}$ However, as the reliability of the examination depends on the experience of the investigator, we performed a preliminary study comparing the results of continuous wave Doppler in 266 carotid arteries and 168 vertebral arteries clearly visualised by transfemoral arterial angiography, performed at our Clinic between 1977 and 1981. When only lesions 
reducing vessel lumen at least by $50 \%$ were considered, the comparison yielded a sensitivity of $91.6 \%$ and a specificity of $95.1 \%$ for the carotid arteries and, respectively, $71.8 \%$ and $82.3 \%$ for the vertebral arteries. We considered a continuous wave Doppler examination as normal when the frontal artery signals were symmetrical and correctly directed, and when the common, internal and external carotid artery signals were all adequately recorded along their cervical course, without any evidence of accelerated flow. ${ }^{15}$ Additionally, vertebral artery flow was recorded at the post-mastoideal slope and at the origin of the artery beneath the supraclavicular fossa..$^{15}$

Digital subtraction angiography was carried out using a $16 \mathrm{G}$ needle, $5 \mathrm{~cm}$ long, placed in an antecubital vein of the right arm. A total of $40 \mathrm{cc}$ of contrast medium followed by $15 \mathrm{cc}$ of $5 \%$ glucose solution were power-injected at the rate of $15 \mathrm{ml} / \mathrm{s}$, for each projection. The standard angiographic study consisted of a left anterior oblique projection of the aortic arch, two oblique projections of the carotid bifurcations, and an anteroposterior projection of the intracranial district. All digital subtraction angiography images were interpreted by a neuroradiologist, who was unaware of the results of continuous wave Doppler examinations. Irregularity was defined as a reduction of less than $10 \%$ of vessel diameter. Symmetrical broadening beyond normal dimensions of the lumen was considered as dilatation. If the vertebral arteries of the same subject showed more than $50 \%$ difference in diameter, they were judged to be asymmetrical. The carotid and vertebrobasilar trees were divided into 10 tracts, as shown in the table.

\section{Results}

Thirty seven patients were males and 13 females. Their mean age was 59 years (range: $43-75$ years). Twenty seven patients presented with TIA and 23 with minor stroke. Forty two patients had attacks involving the carotid artery distribution, whereas eight had vertebrobasilar attacks.

Forty five $(90 \%)$ of our intravenous angiograms have been of technically satisfactory or good quality. In 12 cases $(24 \%)$ digital subtraction angiography showed arterial lesions which had not been detected by continuous wave Doppler. These results, along with the type of lesions found, are reported in the table, where cervico-cranial arteries are divided into ten different tracts. Digital subtraction angiography revealed 14 atherosclerotic dilatations, 10 vesselwall irregularities, eight stenoses affecting between 10 and $50 \%$ of the vessel diameter, two kinkings, 12 osteophytic compressions and one suspected aneurysm, all undetected by continuous wave Doppler. Of the eight stenoses visualised by digital subtraction angiography, six were confirmed at arterial angiography. Three patients could not be submitted to conventional arteriography, two owing to their advanced age and general serious conditions and one with the suspected aneurysm. Discrepancy bet-

Table

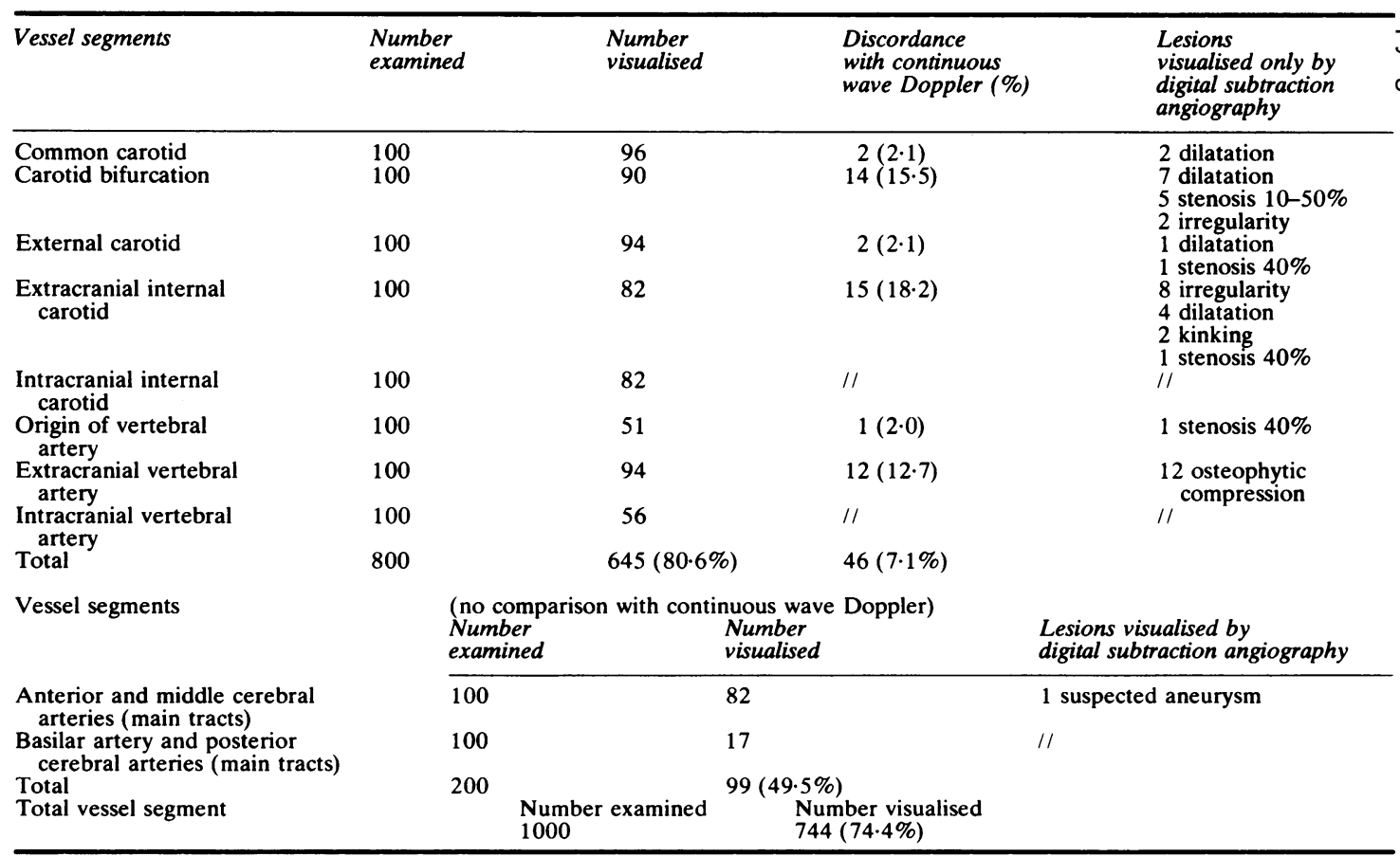


ween continuous wave Doppler and digital subtraction angiography was $7 \cdot 1 \%$ for all visualised arterial tracts. Digital subtraction angiography by itself gave higher visualisation in the extracranial tracts than in the intracranial ones $(84.5 \%$ v. $59.2 \%)$.

Digital subtraction angiography was able to visualise both vertebral arteries in 47 patients (94\%). Of the five cases with vertebral asymmetry demonstrated by digital subtraction angiography, only one had been previously diagnosed by continuous wave Doppler.

Adverse reaction to contrast medium occurred in three patients: two had local erythema at the side of injection. A third patient presented bilateral exophthalmos with retro-orbital pain 15 hours after the examination; symptoms disappeared spontaneously in 48 hours. No neurological complication occurred in any of the patients.

\section{Discussion}

Conventional angiography remains the most accurate diagnostic technique in patients with cerebrovascular disease. Owing to the lower risks which it entails and to the possibility of displaying even minor lesions, digital subtraction angiography has given rise to considerable enthusiasm.

In this study digital subtraction angiography allowed a more sensitive morphological diagnosis in $24 \%$ of patients with previously normal continuous wave Doppler. This figure is apparently in accordance with the results reported by Celesia et al. ${ }^{16}$ In their series, digital subtraction angiography revealed carotid artery lesions in $24 \%$ of patients with normal continuous wave Doppler examination. However, they considered only significant stenoses or occlusions in discussing the comparison between digital subtraction angiography and continuous wave Doppler. In our study, we had no false-negative continuous wave Doppler results, and the discrepancies between the two methods regard only minor, haemodynamically non-significant arterial abnormalities, as well as vertebral artery lesions, which were not considered in that study.

Carotid artery bifurcation is the most frequent site of atherosclerotic lesions; accordingly, the discordance between our two methods was $15.5 \%$, significantly higher than the discrepancy for all other carotid tracts ( $p<0.01$ by chi square test). This finding assumes particular importance if one considers that $10 \%$ of bifurcations were not satisfactorily visualised. Differing anatomospatial positioning ${ }^{17}$ explains why visualisation of the carotid bifurcation was not achieved in 10 cases. It is actually not easy, when positioning the image tube, to anticipate the inclination of the bifurcation so as to obtain a good projection. In fact, the good imaging of the extracranial regions of the internal carotid artery was prevented by superimposition of other arterial tracts.

The relative weakness of the digital subtraction angiography performance in visualisation of the intracranial regions must be taken into account. The figure was $.59 .2 \%$ as compared to $84.5 \%$ for the extracranial regions alone. This weakness had already been noted by other authors. ${ }^{9121819}$ Again, the main problem is superimposition of different vessels, due to simultaneous imaging of all intracranial arteries at the same time. None of the alternative intracranial projections gets round this problem. For our part we find the anteroposterior projection best.

Owing to the possibility of visualising minor arterial lesions, digital subtraction angiography yielded superior results compared to continuous wave Doppler. As even these lesions may be ulcerated and therefore important in the pathogenesis of cerebrovascular attacks, ${ }^{20}$ their detection appears important. However, digital subtraction angiography brought no clear advantage in patients' management. In fact, out of 10 patients with irregularities or minor stenoses of the carotid artery, four were considered to be too old for surgery, and therefore received medical therapy only; in the other six, traditional intra-arterial angiography was necessary in order to evaluate definitely the indication for carotid artery surgery, and it confirmed in every case the minor or mild stenotic lesion (fig.).

We believe that the percentage of the stenotic lesions recorded does not always reveal whether the cerebral ischaemia is due to the stenosis. Some patients with an "insignificant" carotid stenosis can have ischaemic attacks because of the stenosis, and some patients with a "significant" stenosis can have ischaemia caused by heart disease or by intracranial arterial disease. So, because of the continuous wave Doppler inaccuracy to detect less than $50 \%$ stenosis, and because of the digital subtraction angiography inability to offer a complete view of the extra and intracranial arterial tracts, traditional arteriography must be considered to be the definitive examination to decide an endarterectomy.

Lastly, we wish to comment on the study of vertebral arteries by digital subtraction angiography, which has received little attention in previous reports. The main limiting factor was the low visualisation rate of the origins, which are the most frequent site of involvement by atherosclerotic lesions of the posterior circulation. ${ }^{21}$ Only $51 \%$ of vertebral arterial ostia were visualised; this was due to the frequent superimposition of other vessels with the right vertebral artery origin in the left anterior oblique projection. By contrast, vertebral arteries were 

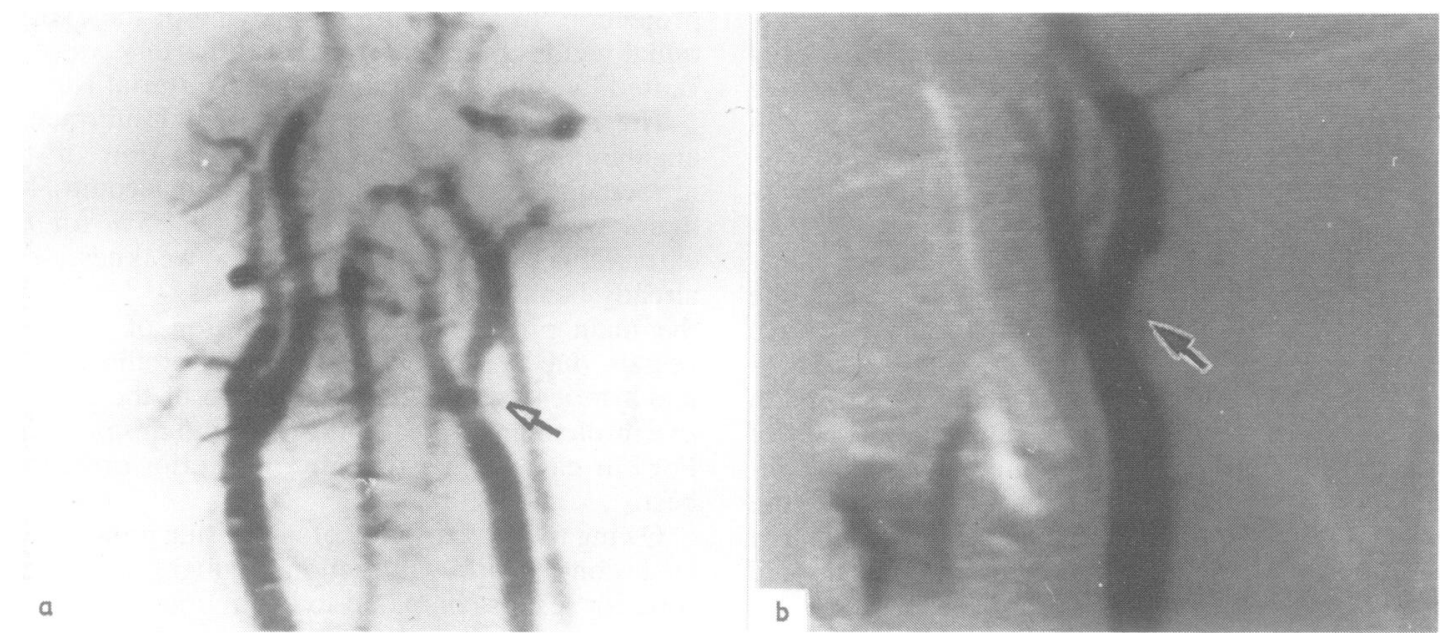

Fig (a) Moderate stenosis of left internal carotid artery at its origin with possible ulcer (arrow). (b) Arterial angiography confirmed the stenosis with suspected ulceration (arrow).

satisfactorily shown along their extracranial course, allowing the clear detection of asymmetries. We conclude that digital subtraction angiography as a diagnostic tool is superior to continuous wave Doppler. However, it is expensive, it entails a minimal but definite risk due to contrast medium injection, and it does not appear, at present, to modify the therapeutic approach of patients with cerebral ischaemia and normal continuous wave Doppler examination.

We thank Mr Luigi Grilli for his assistance in preparing the manuscript.

\section{References}

'Marshall J, Wilkinson IMS. The prognosis of carotid transient ischaemic attacks in patients with normal angiograms. Brain 1971;94:395-402.

${ }^{2}$ Moore WS, Boren C, Malone JM et al. Natural history of non-stenotic asymptomatic ulcerative lesions of the carotid artery. Arch Surg 1978;113:1352-9.

${ }^{3}$ Faught E, Trader SD, Hanna GR. Cerebral complication of angiography for transient ischemic and stroke: prediction of risk. Neurology (Minneap) 1979;29:4-5.

${ }^{4}$ Olivecrona $\mathrm{H}$. Complications of cerebral angiography. Neuroradiology 1977;14:175-81.

${ }^{5}$ Goran A, Moore G. Value of the non-invasive cerebro-vascular laboratory in diagnosis of extracranial carotid artery disease. Stroke 1980; 11:325-8.

6 Weaver RG, Howard H, McKinney WM, Ball MR, Jones AM, Toole JF. Comparison of Doppler Ultrasonography with arteriography of the carotid artery bifurcation. Stroke 1980;11:402-4.
${ }^{7}$ Forbes GS, Earnest DB, Folger WN, Sundt TM. Digital Angiography. Introducing digital technique to clinical cerebral angiography practice. Mayo Clin Pró 1982; 57:683-93.

${ }^{8}$ Little JR, Furlan AJ, Modic MT, Bryerton B, Weinsteir? MA. Intravenous Digital Subtraction Angiography im brain ischemia. JAMA 1982;247:3213-6.

${ }^{9}$ Little JR, Furlan AJ, Modic MT, Weinstein MA. Digita Subtraction Angiography in cerebrovascular disease Stroke 1982;11:557-66.

${ }^{10}$ Turski PA, Strother CM, Turnipseed WD, et ato Evaluation of extracranial occlusive disease by digita subtraction angiography. Surg Neurol 1981;16:394-8.

" Chilcote WA, Modic MT, Pavlicek WA, Little JR, Furlan AJ, Duchesnean PM, Weinstein MA. Digital Subtraction Angiography of the carotid arteries: a comparative study in 100 patients. Radiology 1981;139:287-95.

12 Duff TA, Turski PA, Sackett JF, Strother CM, Crummy A. Evolving role of Digital Subtraction Angiography in neurosurgical practice. Neurosurgery 1982;11:430-8.

${ }^{13}$ Modic MT, Weinstein MA, Chilcote WA, et al. Digital Subtraction Angiography of the intracranial vascular system: comparative study in 55 patients. $A m ~ J$ Roentgenol 1982; 138:299-306.

${ }^{14}$ Trockel U, Hennerici M, Aulich A, Sandmann W. The superiority of combined continuous wave Doppler examination over periorbital Doppler for the detection of extracranial carotid disease. J Neurol Neurosurg Psychiatry 1984;47:43-50.

is Büdingen HJ, von Reutern GM, Freund HJ. DopplerSonographie der extrakraniellen Hirnarterien. Grund lagen - Methodik - Fehlermöglichkeiten - Ergebnisse. Stuttgart, Georg Thieme Verlag, 1982.

${ }^{16}$ Celesia GG, Strother CM, Turski PA, Stieghorst MF, Sackett JF, Mistretta C. Digital Subtraction 
Arteriography. A new method for evaluation of extracranial occlusive disease. Arch Neurol 1983;40: 70-4.

${ }^{17}$ Kaseff LG. Positional variations of the common carotid artery bifurcation: implications for digital subtraction angiography. Radiology 1982;145:377-8.

${ }^{18}$ Carmody RF, Smith JR, Seeger JF, Ovitt TW, Fisher III MD, Capp MP. Intracranial applications of digital intravenous subtraction angiography. Radiology 1982;144:529-34.

${ }^{19}$ Seeger JF, Weinstein PR, Carmody RF, Ovitt TW,
Fisher III MD, Capp MP. Digital Video subtraction angiography of the cervical and cerebral vasculature. $J$ Neurosurg 1982;56:173-9.

${ }^{20}$ Eikelboom BC, Riles TR, Mintzer R, et al. Inaccuracy of angiography in the diagnosis of carotid ulceration. Stroke 1983; 14:882-5.

${ }^{21}$ Hass WK, Fields WS, North RR et al. Joint study of extracranial arterial occlusion: II. Arteriography. Techniques, sites and complications. JAMA 1968;203:961-8. 